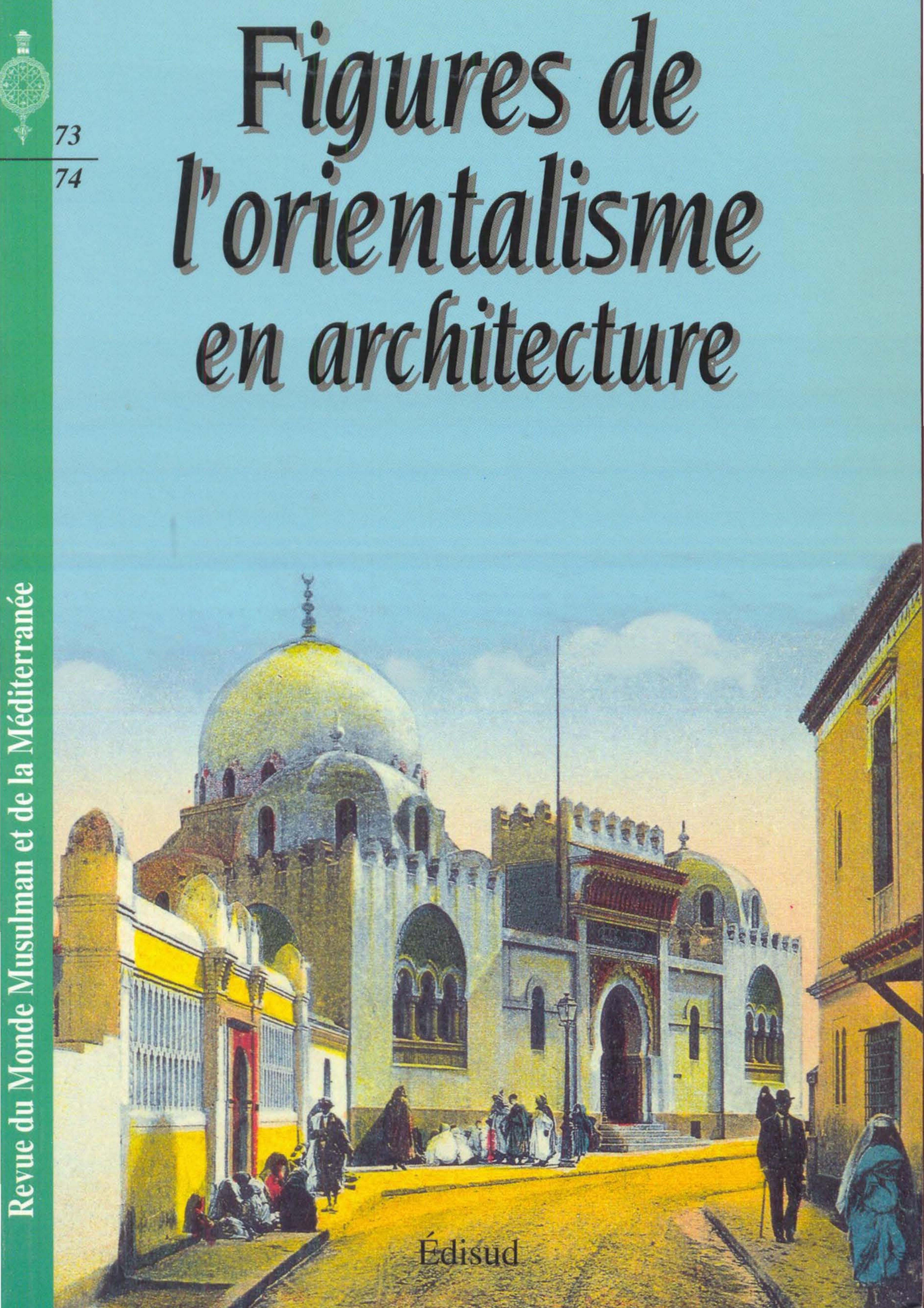


La Revue du Monde Musulman et de la Méditerranée est une publication pluridisciplinaire de l'Association pour l'Étude des Sciences Humaines en Afrique du Nord et au Proche-Orient, réalisée avec la collaboration de l'Institut de Recherches et d'Études sur le Monde Arabe et Musulman.

Paraissant depuis 1966, éditée par les Éditions Édisud depuis 1986, trimestrielle depuis 1987, la revue est publiée avec le concours du Centre National de la Recherche Scientifique (Paris), de l'Université de Provence et du Centre National du Livre.

\section{Comité scientifique}

P. R. Baduel, P. Boyer, G. Camps, S. Chaker, H. Claudot-Hawad, M. Flory, J.-C. Garcin, M. Gast, L. Golvin, J.-R. Henry, R. Ilbert, A. Mahiou, R. Mantran, D. Panzac, A.-L. de Prémare, A. Raymond, G. Souville, N. Sraieb, Ch. Vial.

$\begin{array}{ll}\text { Abonnement } 1994 \text { (4 nos par an) } & 320 \mathrm{FF} \\ \text { Abonnement } 1995 \text { (4 nos par an) } & 350 \mathrm{FF} \\ \text { Abonnement } 1996 \text { (4 nos par an) } & 350 \mathrm{FF}\end{array}$

DIRECTION/RÉDACTION

\author{
Directeur de la Revue \\ Pierre Robert Baduel \\ (CNRS-IREMAM)
}

Directeur-adjoint-trésorier Daniel Panzac (CNRS-IREMAM)

Rédactrice

Viviane Fuglestad-Aumeunier

(CNRS-IREMAM)

\author{
Secrétaire \\ Jany Puccinelli
}

Maison de la Méditerranée

5, avenue Pasteur

13617 Aix-en-Provence Cédex

Tél. 42215988 - Fax 42215275

ÉDITION/DIFFUSION

Éditions ÉDISUD

La Calade, RN 7

13090 Aix-en-Provence

Tél. 42216144 - Fax 42215620

* Anciennement Revue de l'Occident Musulman et de la Méditerranée (du n 1-1966 au n 47-1988).

- Les opinions émises dans les articles ou notes de la Revue n'engagent que la responsabilité de leur (s) auteur (s).

- La Revue n'est pas responsable de la perte des articles, tout auteur est censé conserver l'original de son texte.

- Envoyer le règlement directement au service d'abonnement de l'éditeur.

- Tarif spécial pour envoi par avion.

- Les demandes en duplicata de numéros non arrivés à destination ne pourront être admises de la part des abonnés au-delà de deux mois après publication du numéro suivant de la revue.

- Toute représentation ou reproduction, intégrale ou partielle, faite sans le consentement de l'auteur, ou de ses ayants-droit, ou ayants-cause, est illicite (loi du 11 mars 1957, alinéa 1er de l'article 40). Cette représentation ou reproduction, par quelque procédé que ce soit, constituerait une contrefaçon sanctionnée par les articles 425 et suivants du Code pénal. La loi du 11 mars 1957 n'autorise, aux termes des alinéas 2 et 3 de l'article 41, que les copies ou reproductions strictement réservées à l'usage privé du copiste et non destinées à une utilisation collective d'une part et, d'autre part, que les analyses et courtes citations dans un but d'exemple et d'illustrations. 
Antonio Bravo Nieto*

\section{La genèse d'un style colonial : l'architecture rifaine dans le Maroc espagnol}

Cet article se propose d'analyser un modèle d'architecture lié au fait colonial et qui fut élaboré dans une des régions du Protectorat espagnol du nord du Maroc (le Rif) au cours des années 1930-40 : "l'architecture rifaine". Mener à bien une telle analyse exige d'examiner tout d'abord quelques unes des circonstances dans lesquelles devait émerger cette architecture, et donc de tenter de définir "les sources politiques et culturelles de l'outre-mer" qui ont nourri l'imaginaire esthétique de la production artistique espagnole en Afrique du Nord. Elle exige également de s'intéresser au profil professionnel de celui qui en fut le principal maître d'œuvre : Emilio Blanco de Izaga, officier des Intervenciones Militares (Interventions militaires) dans le Protectorat marocain.

\section{Les sources politiques et culturelles du style rifain}

On peut affirmer que les Intervenciones Militares et le berbérisme ont respectivement représenté le cadre politique et culturel qui devaient donner naissance à l'architecture rifaine ; on ne saurait en effet négliger à cet égard qu'Emilio Blanco de Izaga devait passer d'un modeste poste d'Interventor Militar (contrô-

* Historien de l'architecture et de l'urbanisme, enseignant à l'UNED, centre de Melilla. 
leur militaire) dans un hameau reculé du Rif à celui de délégué des Affaires indigènes du Haut Commissariat, et qu'il fut sans conteste un des plus brillants berbéristes espagnols.

La section des Affaires indigènes joua un rôle fondamental dans les mécanismes que l'Administration espagnole utilisa pour assurer le contrôle de la société marocaine, et le contrôleur fut son fonctionnaire le plus marquant. Ce dernier appartenait à un corps de volontaires constitué par des officiers de l'Armée ${ }^{1}$ et qui était organisé de façon pyramidale.

Dans chacune des Régions du Protectorat, on trouvait ainsi un contrôleur territorial dont les fonctions couvraient, entre autres, le contrôle des entités municipales, l'administration politique, la sécurité et l'ordre public, le contrôle de la Mejaznia (police indigène), la santé et la bienfaisance, etc. Ce contrôleur territorial (ayant grade de lieutenant-colonel) exerçait son commandement depuis un Bureau central dont dépendaient les différents Bureaux de district (sous le commandement d'un capitaine) ; de ceux-ci dépendaient à leur tour d'autres Bureaux auxiliaires d'Information (ayant à leur tête un lieutenant-contrôleur). Le tout constituait un réseau dense de contrôle politique et social couvrant la totalité du territoire, et ce jusqu'aux plus petits douars.

Le contrôleur appartenait à un corps dont l'importance avait été pressentie par des personnalités influentes engagées dans la colonisation espagnole. Ainsi celui qui devait plus tard devenir le Chef de l'Etat espagnol, Francisco Franco Bahamonde (1926 a, 121 et 1926 b, 265-266), s'exprima-t-il à maintes reprises sur le rôle que ce corps devait jouer dans la réorganisation régionale que la métropole se proposait d'entreprendre dans le Rif récemment pacifié. Pour ce dernier, les contrôleurs devaient devenir la " cellule de base de l'organisation politique ", et c'est pour cette raison qu'il était indispensable de les préparer par des cours spéciaux afin qu'ils puissent mener à bien une solide œuvre de colonisation.

Les fonctions des contrôleurs furent multiples, mais les plus caractéristiques furent centrées sur l'information, qu'une fois obtenue, ils se devaient de transmettre à leurs supérieurs : « les contrôleurs sur le terrain sont les yeux et les bras du commandement " (Casas Morá, 1948, 153). Pour pouvoir "intervenir", il fallait ainsi connaître, comprendre, être parfaitement informé de tous les aspects qui constituaient la vie des kabilas ${ }^{2}$, villes, sociétés et cultures sous intervention. Le contrôleur se devait donc d'être un parfait connaisseur de la réalité sociale, culturelle et politique du lieu où il exerçait son métier. Il n'est ainsi guère étonnant qu'un tel poste ait été volontairement sollicité par des officiers séduits par le caractère fascinant du monde marocain et attirés par l'enquête sociologique, les us et coutumes ou la langue. Le fait est que de ce corps allait jaillir tout au long des années 1930 et 1940 une pléiade de véritables chercheurs, dont la production écrite ${ }^{3}$ et architecturale en ses formulations esthétiques est pour nous du plus grand intérêt.

Bien des contrôleurs militaires furent en effet amenés à faire œuvre d'architecture, même si le fait est paradoxal puisque cette fonction fut exercée en marge des deux entités qui, en théorie, en avaient la compétence exclusive : le corps des Ingé- 
nieurs de l'Armée et le service d'Architecture et des Travaux Publics du Haut Commissariat. En raison de la spécialisation fonctionnelle obligée des ingénieurs militaires 4 , et du personnel réduit du Service d'Architectures, le contrôleur eut ainsi à assumer certaines responsabilités comme la réalisation de bureaux et autres annexes liées aux activités d'intervention, de casernes pour les forces de Mehala (troupes indigènes) ainsi que des constructions reconnues d'utilité publique, tels que des abattoirs, écoles, mosquées, sanctuaires, fontaines, puits et abreuvoirs, guérites de surveillance, ponts, etc.

En théorie, le contrôleur ne devait veiller qu'à l'exécution du chantier, selon le projet en principe fourni par les techniciens du service d'Architecture. Mais dans la pratique, les constructions se trouvèrent le plus souvent placées sous leur responsabilité exclusive : étant donnée l'extrême dispersion des zones de peuplement, ces techniciens n'y arrivaient pas toujours à temps (voire même jamais) et le contrôleur devait alors décider par lui-même. Ce fait est encore plus particulier si nous examinons le corps militaire d'origine de la plupart des contrôleurs ; contre toute attente, une grande partie d'entre eux provenait de l'infanterie et rares étaient ceux (en 1929, par exemple, ils n'étaient que deux) qui possédaient le titre supérieur de l'Académie des ingénieurs de l'armée de Guadalajara et avaient reçu une formation technique ou académique les préparant à la mise en œuvre de travaux de construction.

Tout porte à croire que les missions exercées par ces contrôleurs professionnels aux pouvoirs quasi illimités dans leur circonscription n'allèrent pas sans poser quelques problèmes : se livrant à l'occasion à un penchant excessif pour la réalité qu'ils devaient contrôler, certains firent surtout fonction de directeurs de travaux de construction qui, quoique mineurs, avaient néanmoins leur importance. Ainsi les contrôleurs furent-ils mis en garde du danger qu'ils encouraient de devenir "d'admirables chercheurs" (Casas Mora, 1948, 154), tandis qu'en d'autres occasions les excès esthétiques de leurs constructions furent censurés. Tout aussi explicite est l'avertissement donné en 1950 par le délégué des Travaux Publics, Vicente Martorell Otzet : les contrôleurs devaient certes être un peu architectes et ingénieurs, mais éviter de "s'enivrer dans la construction" (Martorell Otzet, 190-193); pour ce faire, ils devaient obligatoirement toujours laisser les questions esthétiques aux architectes et chacun devait en outre éviter de créer un style personnel dans l'exercice de sa charge 6 . Ces allusions, proférées à la fin des années 1940 , étaient destinées à réprimer les architectures individuelles mais elles nous éclairent aussi sur le fait quauparavant, c'est tout le contraire qui s'était produit.

La deuxième réalité à prendre en considération pour l'étude des racines de l'architecture d'Emilio Blanco est le berbérisme ou « rifainisme ", phénomène culturel qui englobe tout un courant général d'intérêt envers la société rifaine, ses coutumes, sa culture. Ce phénomène avait déjà commencé à se manifester à l'occasion des premiers contacts (ou rendez-vous manqués) des espagnols avec le Rif mais, en tant que phénomène culturel, il tendra plus particulièrement à s'affirmer à partir des années 1920 pour atteindre son apogée à la fin des années 19407. Ce 


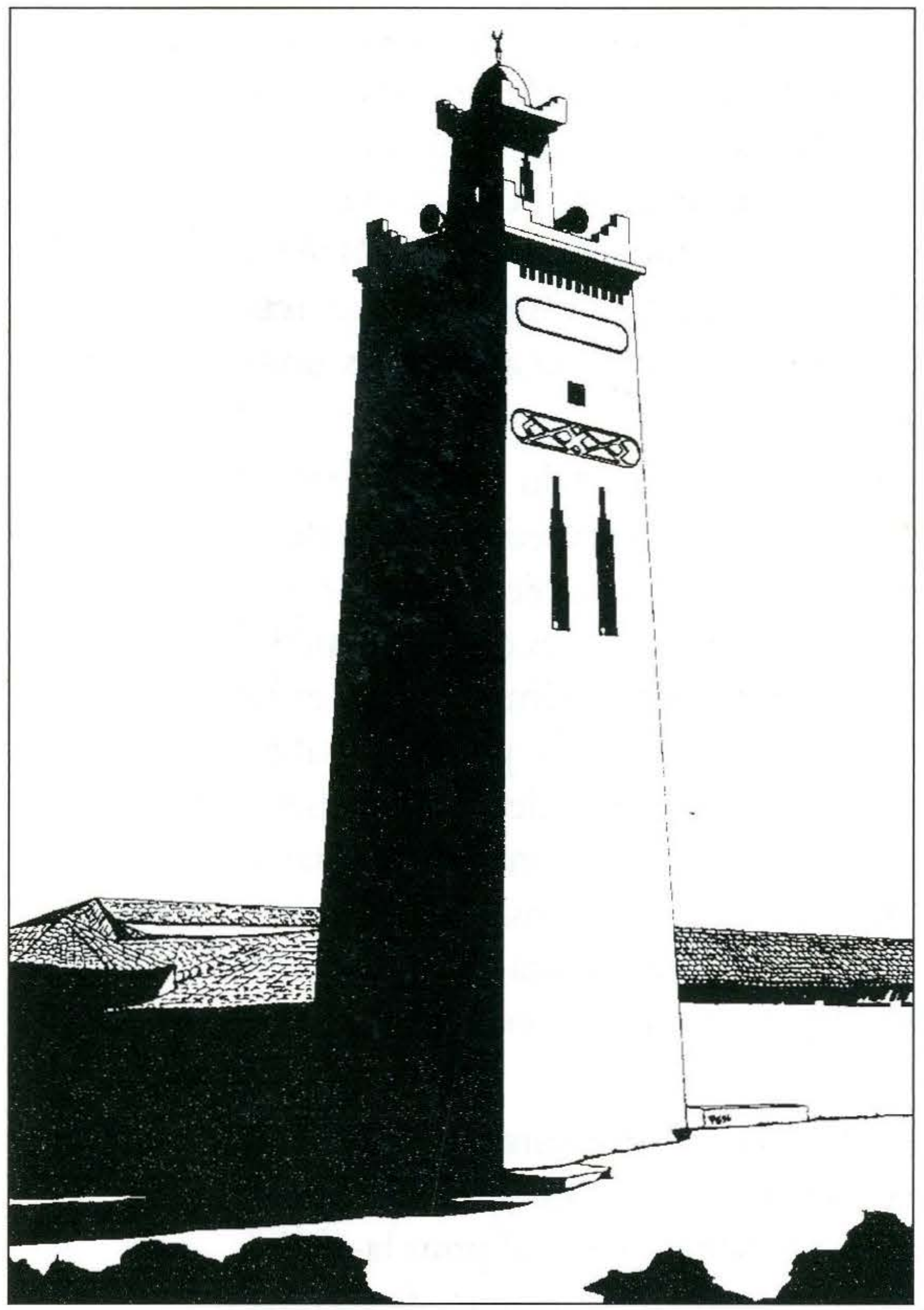

Minaret de la mosquée de Axdir, en 1991.

Restitution : Pascal Carret.

"peuple rifain" suscitait des passions contrastées dans la métropole ; toutefois, quelle que fût l'attitude prise, celle-ci était toujours radicalement influencée par la sanglante guerre anti-coloniale que les rifains menaient depuis 1921 contre l'armée espagnole. Ainsi, si dans l'opinion espagnole, les rifains étaient généralement perçus comme un peuple arriéré et soumis à une décadence ancestrale dont il fallait le tirer, cette idée n'effaçait pas les sympathies que pouvait éveiller cette "race" supposée indépendante et fière ${ }^{8}$. Telle était aussi l'opinion de Francisco Franco (1925) lorsqu'il écrivait que la culture berbère appartenait à une civilisation ancestrale digne d'admiration et de respect, mais qu'il fallait la transformer lentement par la voie de l'éducation?.

\section{Emilio Blanco de Izaga et l'architecture rifaine}

Emilio Blanco de Izaga (Orduña-Bilbao 1892 - Madrid 1949) apparaît déjà comme contrôleur à Punta Pescadores en avril 1927 ; trois ans plus tard, il écrivait son premier livre sur l'habitat traditionnel rifain (1930) avant d'être affecté dans une autre kabila du Rif, Ait Ammart. En 1932, il est nommé à Izimmuren ; il sera deux ans plus tard promu contrôleur de district à Axdir. A partir de ce moment, il fera rapidement carrière au Maroc : en 1936, il est contrôleur territorial du Rif, poste qu'il conservera jusqu'en 1942 ; il effectue alors un court séjour en Guinée Equatoriale, puis il est nommé cette même année sous-délégué des Affaires Indigènes et obtient finalement, le 8 août 1944 (alors qu'il atteint le grade de lieutenant colonel), le poste de délégué des Affaires Indigènes au Haut Commisariat de l'Espagne au Maroc. Il occupera ce poste jusqu'au 10 août 1945, date à laquelle prend fin son influence sur les affaires africaines.

Emilio Blanco vécut une période cruciale de la colonisation du Rif en raison des transformations profondes qui commençaient à s'opérer dans sa société et sa culture ; toujours est-il qu'il ne joua pas un rôle passif d'observateur et devait tenter, pendant les dix-huit années durant lesquelles il fut contrôleur, d'exercer une influence directe allant dans le sens de ces changements. 
L'œuvre d'Emilio Blanco s'est développée dans trois champs très étroitement liés : une activité politique de contrôleur ; une œuvre écrite sur la société rifaine (Hart, 1958 et 1975) et un travail dans le domaine de l'architecture (Sierra Ochoa, 1951 ; Bravo Nieto, 1991). Son œuvre ne fut pas toujours bien comprise de ses supérieurs (Hart, 1975, 22), même si ce fait semble être, en principe, en contradiction avec les postes de responsabilité qu'il occupa. Emilio Blanco croyait fermement qu'il fallait agir sur la société rifaine car elle lui apparaissait (ainsi qu'il l'écrivait en décembre 1935) comme "une anarchie organisée" (Hart, 1975, 62), exposée à sombrer dans la violence étant donné son profond état de décomposition sociale (Blanco Izaga, 1939, 9 et 18). Mais il estimait surtout inopportune l'implantation de formes arabes de contrôle sur une société berbère ; c'est aussi pour cette raison qu'il prétendit ressusciter le droit coutumier rifain (Hart, 1975,21 ) en entreprenant de le codifier en vue d'une application ultérieure. Or ce point de vue n'entrait nullement dans les vues des autorités espagnoles, puisqu'il revenait à diviser le Protectorat en deux zones différentes, l'une arabe, l'autre berbère ${ }^{10}$.

\section{Le style rifain: un style officiel?}

Il est évident que l'architecture rifaine naquit dans un contexte tout à fait propice à son expansion ; après la pacification régionale, une vaste politique de construction s'était en effet avérée nécessaire pour pallier l'éparpillement absolu des zones de peuplement et des habitations. Cette dispersion était considérée par les autorités espagnoles comme un atavisme et donc comme quelque chose à corriger (Franco Bahamonde, 1925, 6) en encourageant l'urbanisation ou en développant les chefferies. Le travail d'Emilio Blanco s'inscrit ainsi dans une politique globale de construction, mais le fait à souligner est que sa formulation esthétique de l'architecture rifaine fut plutôt tolérée que mise en avant par les autorités du Haut Commissariat qui ne voyaient dans la question d'un nouveau style que l'excentricité individuelle d'un militaire d'une valeur, cela étant, indiscutable. Alfonso de Sierra Ochoa (1951) a déjà suggéré une possible genèse politique de l'architecture rifaine ${ }^{11}$. Nous avons, pour notre part, souligné par ailleurs (Bravo Nieto, 1991, 270-272) que cette architecture fut un style officiel parce qu'il fut mis en œuvre par l'administration espagnole et put se déployer régionalement sans problèmes ni entraves majeurs, mais qu'il ne fut nullement un style reconnu comme tel par les instances politiques, ni même dûment diffusé ou prôné à son époque. En outre, si on s'en tient à la copieuse littérature africaniste qui inonda l'Espagne franquiste, on en concluerait que ce style n'a jamais réellement existé12.

Il est vrai que les bases sur lesquelles fut bâtie l'architecture rifaine furent très circonstancielles, par trop sujettes à une inspiration et à une formation purement individuelle et qu'elles ne dépassèrent pas la période durant laquelle Emilio Blanco résida au Maroc. Il est tout aussi significatif, en ce sens, que ce style 
n'ait connu ni continuateurs ni même imitateurs, avant que l'indépendance du Maroc en 1956 ne vienne introduire une rupture avec tout style du passé colonial. Si Emilio Blanco fut un militaire certainement respecté, son style rifain ne reçut pas la reconnaissance publique prévisible : ce fut un style officiel sans existence officielle au delà des frontières du Rif.

\section{Formation et sensibilité artistique de Blanco}

Une interrogation préalable à l'analyse de l'architecture rifaine consisterait à signaler quelles furent la formation artistique et les bases culturelles avec lesquelles pouvait compter ce militaire basque à l'heure de formuler un nouveau style architectural. Rien dans sa biographie professionnelle ne paraît l'y avoir destiné. Blanco appartenait au corps d'Infanterie (comme nombre de contrôleurs) et avait effectué ses études militaires à l'Académie d'Infanterie de Tolède, où il était entré en 1910 ; au cours des années 1920, il enseigna à l'Ecole Centrale de Gymnastique. N'étant pas ingénieur de l'armée, il ne disposait donc d'aucune formation technique ou académique préalable. Sa formation fut en conséquence celle d'un autodidacte, d'une personnalité soucieuse et obsédée par les différentes facettes du Rif : sociales, culturelles, politiques et artistiques. La façon dont Emilio Blanco percevait la réalité rifaine ne provenait pas d'une formation littéraire ; il était même un critique implacable des écrivains orientalistes qui se servaient de clichés dans leurs œuvres (Blanco Izaga, 1930, 5-6), revenaient à des thèmes récurrents et élaboraient leurs essais en se basant exclusivement sur ce qu'avaient écrit leurs prédécesseurs, coutumiers des mêmes agissements en leur temps ${ }^{13}$. Son système de recherche et de travail s'appuyait tout au contraire sur le travail de terrain. Il n'a cessé de souligner dans toutes ses œuvres que la seule formule possible pour appréhender les choses était de se baser sur le contact direct avec la réalité, sur l'annotation directe et sur l'enquête au sens des anthropologues.

Par ailleurs, les "perceptions" de Blanco se sont concrétisées de maintes façons. Nous savons qu'il réussissait avec aisance des aquarelles sur des thèmes marocains. Il illustrait lui-même ses articles avec des dessins au fusain et à l'encre et y faisait montre d'une bonne capacité à capter le mouvement ainsi que d'une stylisation (surtout dans ses multiples et audacieux dessins féminins) très en vogue dans la facture picturale art déco. Son opinion sur un des meilleurs peintres espagnols de l'école orientaliste, Mariano Bertuchi, est très significative : Blanco écrivit en effet qu'il ne s'intéressait ni à sa technique, ni à sa maittrise mais à sa manière de façonner cette réalité marocaine qui les obsédait tous deux (Blanco Izaga, 1946, 315-316). Il s'identifiait avec le processus de perception subjective de Bertuchi, mais il pouvait tout autant extraire d'un tableau l'impression qui avait rendue possible son exécution.

Autodidacte, pourvu de qualités artistiques, doté d'un sens aigü de l'observation et sachant mieux encore la restituer visuellement, Emilio Blanco fut un excellent sociologue mais avant tout un enthousiaste du Rif : voilà ce qui lui tint 
lieu de formation à l'heure où il affirma son projet de création d'un nouveau style architectural.

\section{La genèse du style rifain}

Cette genèse fut la conséquence directe d'un processus de phagocitage d'images mené à bien par Emilio Blanco à partir d'une vaste série d'options culturelles et de courants esthétiques de son temps. En ce qui concerne l'architecture, il put trouver au Maroc espagnol (Tétouan et Larache) une gamme variée de modèles néoarabes, et en particulier ceux qui avaient dépassé une première phase plus archéologisante de ce style et qui dans les années 1920 révélaient une influence directe de l'architecture réalisée au Maroc français par Henri Prost ${ }^{14}$. Dans la ville nordafricaine de Melilla ${ }^{15}$, les modèles architecturaux dont Blanco put avoir connaissance étaient radicalement différents et très liés à des courants européens plus cosmopolites, tel que l'art nouveau d'origine française et italienne et la Sécession viennoise (par Catalogne interposée) jusqu'au milieu des années 1920 (Bravo Nieto, 1993) avant que ne commencent à apparâttre, dès le début des années 1930, les modèles art déco, aussi bien dans les formes les plus cosmopolites dérivées de l'exposition parisienne de 1925, que dans celles plus aérodynamiques redevables à l'esthétique du machinisme avec un soupçon d'expressionisme à la Mendelssohn.

Blanco rejetta le style néo-arabe plus ou moins cubiste et semi-officiel qu'il avait trouvé à Tétouan ou à Larache, tout comme le monde art déco cosmopolite qu'il avait pu découvrir à Melilla ${ }^{16}$ au début des années 1930, car il les considérait tous deux peu appropriés à un contexte berbère; ils lui servirent toutefois de référence et de point de départ pour pouvoir élaborer son architecture rifaine, initiant dès lors une tendance différente et plus originale. Son choix représenta une "troisième voie» sans doute liée au contexte art déco, mais qui s'inscrivait aussi dans un courant qui cherchait à récupérer les esthétiques de civilisations lointaines et exotiques, de peuples et de cultures ancestrales dont les formes devaient être appropriées et assimilées par l'Occident (Pérez Rojas, 1990, 418). Il s'agissait d'un courant généralisé de retour hétérodoxe à l'exotisme, adoptant divers points de vue et donnant lieu à différentes appropriations-réinterprétations. Ce phénomène hétérodoxe englobait les différentes révisions de l'architecture aztèque ou maya aussi bien que la démarche néo-pharaonique (Volait, 1988 et 1992) tout comme ce qui fut appelé l'architecture néo-soudanaise (Leprun et Sinou, 1984, 292). Dans le contexte colonial hispanique, il devait également donner lieu à maintes tentatives significatives de récupération de formes vernaculaires d'architecture, comme celles menées à bien au Sahara Espagnol, à Sidi Ifni, et surtout au Rif.

Un des principaux problèmes qu'Emilio Blanco dut affronter à l'heure de formuler son style fut de trouver des modèles adéquats. Il n'est pas surprenant que sa première œuvre écrite ait traité de l'architecture rifaine vernaculaire et que ses conclusions à ce propos aient été très négatives (Blanco Izaga, 1930, 9)17. Il considérait les constructions populaires rifaines édifiées dans le monde rural 
comme des habitations rustiques, mal bâties et dont il paraissait difficile d'extraire le moindre modèle esthétique notable ${ }^{18}$. Emilio Blanco possédait au préalable une bonne culture architecturale ; dans ses premiers écrits, on trouve ainsi quelques références à ce que lui suggéraient bon nombre de constructions rurales, telles certaines maisons du cercle de Xaona, dans la kabila de Beni Ammart, où il croyait voir un « logement sous-marin » puisque ses « lignes droites rappellent ces modernes navires »; une guérite lui suggérait même le sommet d'un périscope, tandis que dans d'autres bâtiments il croyait trouver en revanche des " maisons à reminiscence égyptienne telle celle de Mohamed Soliman à Tafersit » (Blanco Izaga, 1930, 10-11).

Mais c'est dans les villages berbères du Sud marocain, dans cette architecture de chateaux ou casbahs en terre rouge et de greniers collectifs (Tighrem) dont les lignes nettes et les volumes massifs le séduisirent immédiatement, que Blanco devait finir par trouver les modèles qu'il cherchait avec acharnement. Le puissant effet visuel de ces édifices, leur symétrie, leurs tours d'angle aux profils en talus lui servirent pour élaborer sa proposition formelle ${ }^{19}$. Blanco avait définitivement trouvé la source d'où jaillirait son architecture ; le résultat en fut une production qui n'était plus une récupération des langages esthétiques du passé rifain lui-même, mais le fait d'une "transplantation" de formes, certes tirées du monde berbère mais en marge d'une quelconque référence à l'art arabe.

\section{La responsabilité de l'architecture rifaine}

Le manque de formation technique d'Emilio Blanco se traduisit nécessairement par un type de paternité indirecte sur ce que nous appelons ses œuvres. Ce fut certes lui qui établit la définition formelle de cette architecture, créant ainsi un style qui fut par la suite intégré par toute une série de professionnels qui l'appliquèrent dans leur pratique architecturale. Reste la question essentielle de la responsabilité définitive de ces œuvres, car si dans certains cas il imposa personnellement et sous son inspiration directe les caractéristiques individuelles de quelques édifices (les bureaux d'Intervention de l'Arba de Taurirt, par exemple), ceci ne sera évidemment pas le cas de l'architecture rifaine dans son ensemble. Ce style était donc un modèle obligatoire pour un certain type de constructions; depuis son poste de contrôleur de District, Emilio Blanco exerçait d'ailleurs un contrôle très strict afin que ses suggestions, ses modèles et ses perceptions soient fidèlement suivies par les personnes en charge de la construction, par le biais de dessins ${ }^{20}$, croquis, indications verbales sur le chantier, etc. et c'est aussi par ce biais que fut assurée la diffusion du style rifain.

Emilio Blanco sut influencer et imposer à plus ou moins grande échelle ses critères aux professionnels qui assumèrent la responsabilité technique des travaux. Dans certains édifices ruraux peu importants, cette imposition formelle s'exerçait directement sur l'entrepreneur des travaux ${ }^{21}$, mais dans des chantiers de plus grande envergure, celle-ci s'exerçait sur les architectes du service d'Architecture 
ou sur les ingénieurs des Ponts et Chaussées du service des Travaux Publics et enfin, dans les ouvrages à caractère militaire, sur les ingénieurs de l'armée.

Francisco Hernanz Martínez fut l'architecte en charge des constructions de la région orientale du Protectorat d'octobre 1929 au milieu de 1936, soit au cours d'une période cruciale pour la formulation de l'architecture rifaine. La relation entre Emilio Blanco et Francisco Hernanz, en ce qui concerne la formulation de modèles, est très difficile à préciser, bien que des indices évidents dans certaines œuvres montrent qu'elle ne fut pas sans effet ${ }^{22}$. Après le départ de Francisco Hernanz de la région orientale, ce fut le service des Constructions militaires qui prit en charge toutes les constructions. Plus tard, de mars 1938 à juin 1943, on engagea temporairement l'architecte Manuel Latorre Pastor, dont la position professionnelle fut bien plus instable que celle de son prédécesseur, et qui de ce fait fut nécessairement plus attentif aux exigences formelles de celui qui, à ce momentlà, était déjà contrôleur régional du Rif.

En revanche, le principal collaborateur d'Emilio Blanco, celui avec lequel il travailla étroitement à l'élaboration de plusieurs œuvres, fut un ingénieur des Ponts et Chaussées, Francisco Castellón Díaz. Il est très intéressant de constater la symbiose professionnelle existant entre Blanco et un ingénieur très éloigné en principe des exigences esthétiques conventionnelles ; ce choix est en effet très significatif en ce qu'il met en lumière son éloignement du monde académique au bénéfice de la liberté de style. Le type d'ouvriers qui travailla à cette architecture rifaine dépendait des spécialisations requises : il s'agissait habituellement de maçons et de manœuvres d'origine espagnole en provenance soit du Protectorat (Villa Sanjurjo), soit de Melilla et dont on exigeait une parfaite maîtrise professionnelle. La population rifaine ne parait avoir été employée qu'occasionnellement, à l'exception des cas où aucune qualification ne s'avèrait nécessaire ; ceci se pro-

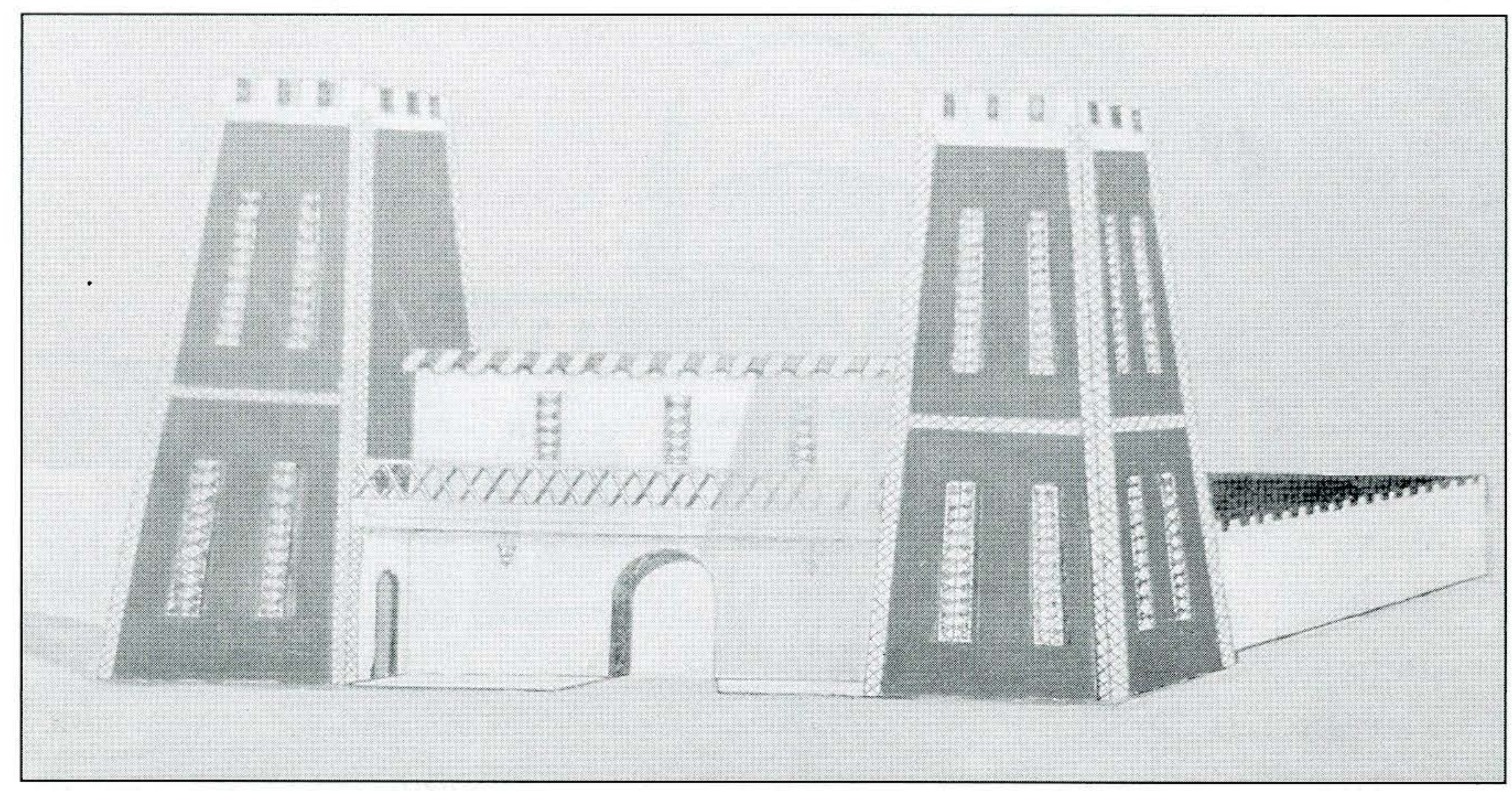

Dessin d'un projet de guérite pour la $102^{e}$ division, région du Rif, sans date ni auteur. Source: Archives du Génie, Melilla, Section technique, su. 
duisit par exemple en 1947 lorsqu'on fit appel à 18200 chômeurs rifains pour la réfection des routes.

\section{Valorisation formelle}

Mise en œuvre chronologiquement dès les premières années des années 1930, et jusqu'au milieu de la décennie suivante, l'architecture rifaine peut être définie comme un savant assemblage de masses et de volumes composé de façon à ce que l'ornementation y soit toujours subordonnée et que la couleur y acquière un caractère particulièrement significatif. Emilio Blanco concevait les espaces architecturaux comme une somme de volumes prismatiques et quadrangulaires composés de manière totalement asymétrique afin d'obtenir un important contraste visuel. C'est le cas de certaines de ses réalisations, telle la mosquée d'Eimzourem, où il rompt avec toute idée de plan conventionnel, en disposant deux tours totalement asymétriques dans la façade principale : jouant un rôle déterminant par leur volumétrie, ces tours participent d'une composition réellement avant-gardiste.

Dans les bureaux de l'Arba de Taourirt (1940-1944), on retrouve cette même composition complexe puisque l'ensemble adopte la forme d'un espace géométrique où alternent des corps flanqués de tours et des espaces construits : c'est sans aucun doute l'œuvre la plus aboutie de Blanco et celle où il pousse au plus haut niveau ses idées sur la combinaison des volumes, de la couleur et de la décoration. Les tours de l'Arba de Taourirt (surtout la plus grande) 23 assument une fonction

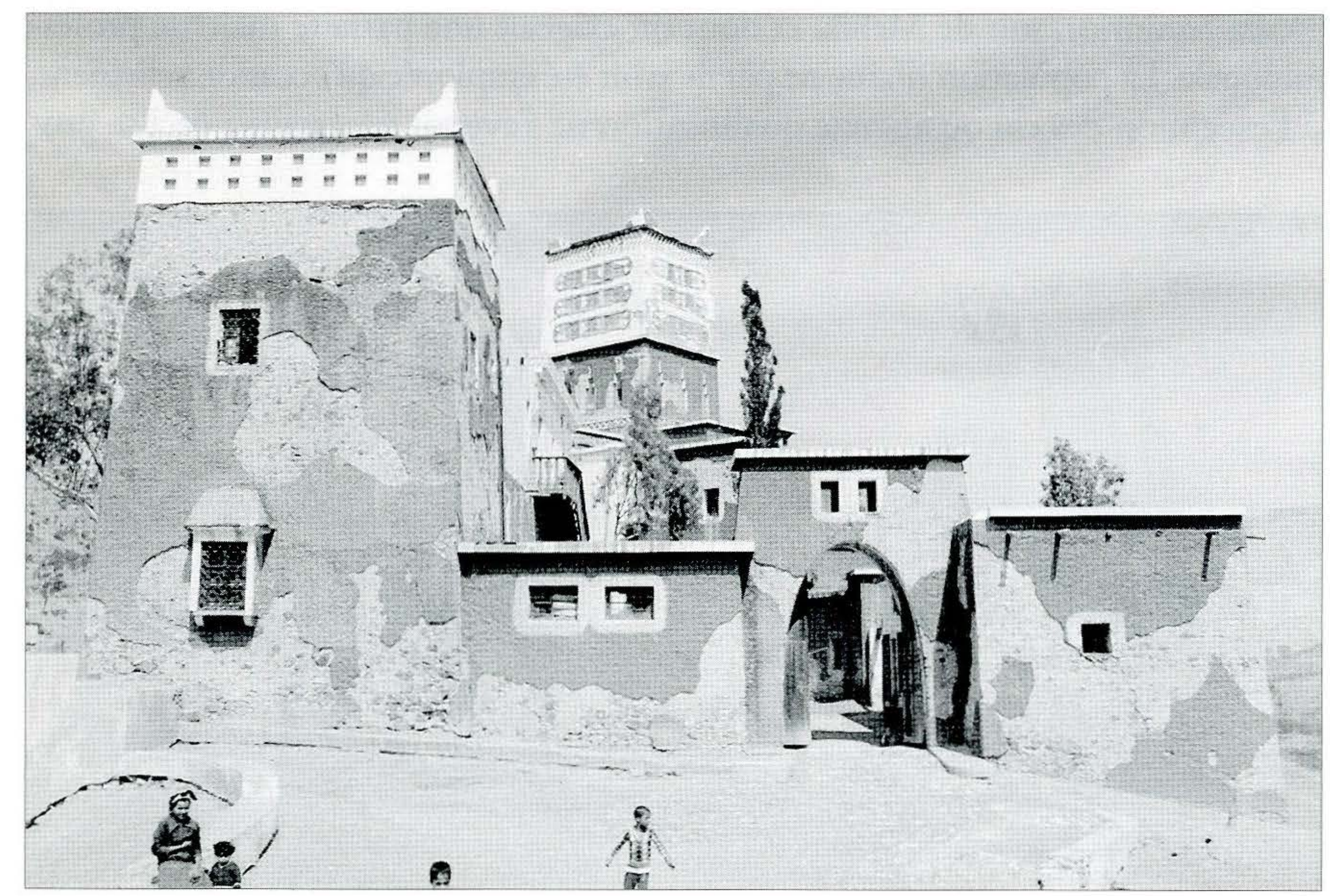

Bureau des Intervenciones militares de l'Arba de Taurirt en 1989. Photographe : Dionisio Hinojo Sánchez. 
de signalisation, à la manière de ces phares futuristes si en vogue pendant la période art déco. Cette image de la tour comme élément générateur est fondamentale dans l'architecture rifaine et elle a très souvent représenté le trait définitif qui donnait du caractère à l'œuvre : tel fut le cas des mosquées d'Axdir, de Sammar Amar den Snada et aussi en quelque sorte de celle d'Alhucemas. Leur minaret prend la forme d'une tour carrée et massive, dont les parois présentent le talus caractéristique qui les rendait larges à la base et stylisées au sommet. L'ornementation de ces tours était effectuée à base de cartouches disposés en bandes horizontales (qui rappellent l'écriture hiéroglyphique égyptienne) avec des signes géométriques, des triangles, des croix en $\mathrm{X}$ et des losanges, réalisés par cisellement du mur et soulignés par des touches chromatiques.

Lidée de masse est fondamentale dans toute l'architecture rifaine : Blanco concevait les édifices comme des masses en talus qui ne sont pas sans évoquer certains monuments funéraires de la civilisation égyptienne ou de l'art maya. Ses compositions incluaient ainsi une sorte de tour trapue, toujours placée latéralement, ainsi qu'on peut l'observer dans certains bâtiments officiels d'Alhucemas, surtout dans un petit tribunal. On retrouve cette même caractéristique dans la station d'adduction d'eau d'Alhucemas, œuvre d'un fort contraste chromatique et volumétrique, et même dans d'autres constructions où une tour ne se justifiait guère et ne jouait qu'un rôle négligeable : c'est le cas de l'abattoir d'Eimzourem. Ailleurs, comme dans le cas de l'orphelinat de filles de Tamasint, c'est l'édifice tout entier qui prend la forme d'un corps en talus, flanqué de tours, et à développement essentiellement horizontal, souligné par une large galerie percée de

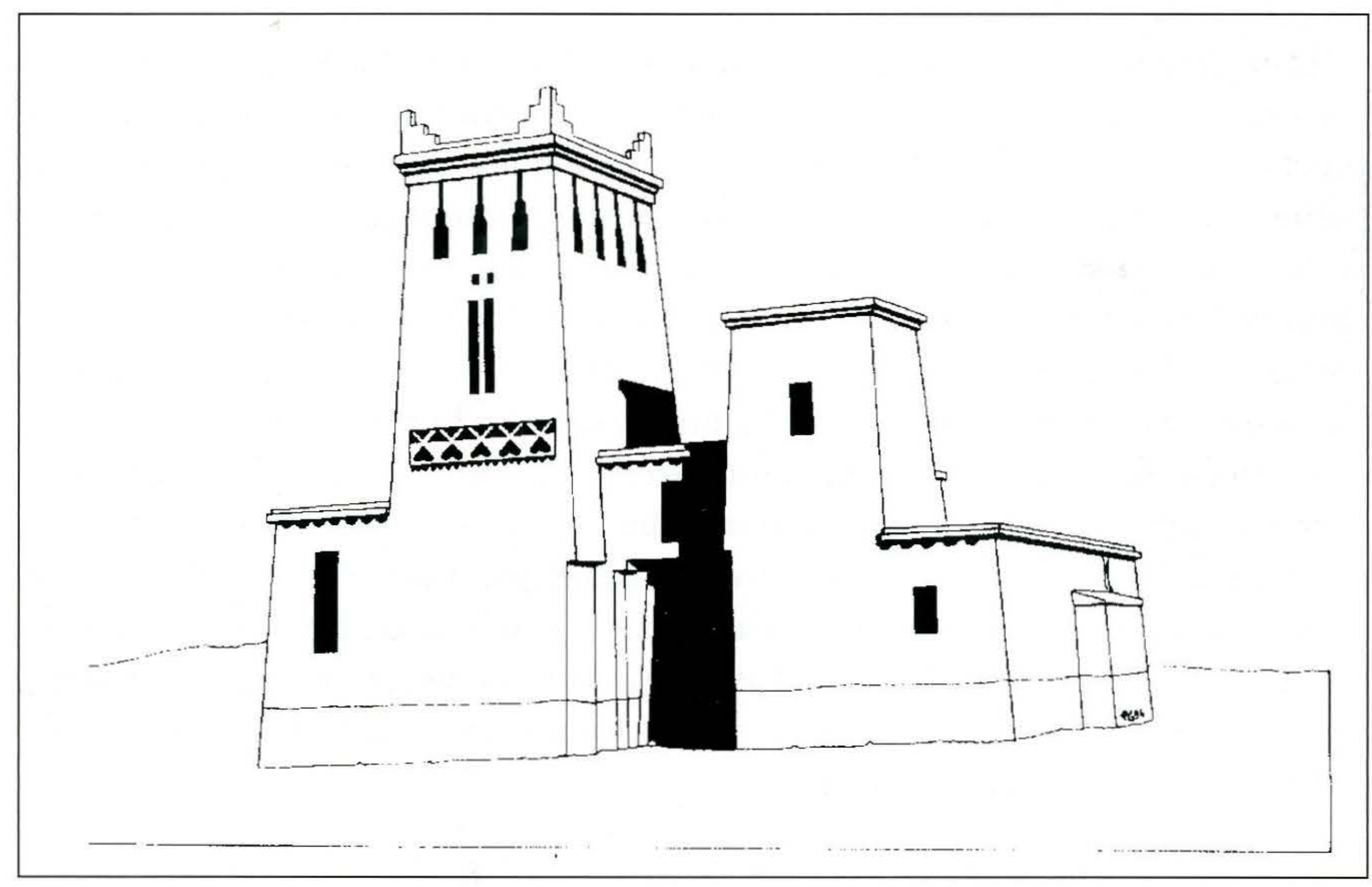

Mosquée de Eimzourem. D’après Mundo (Madrid), n 96, 8 mars 1942, p. 388. Restitution: Pascal Carret. 


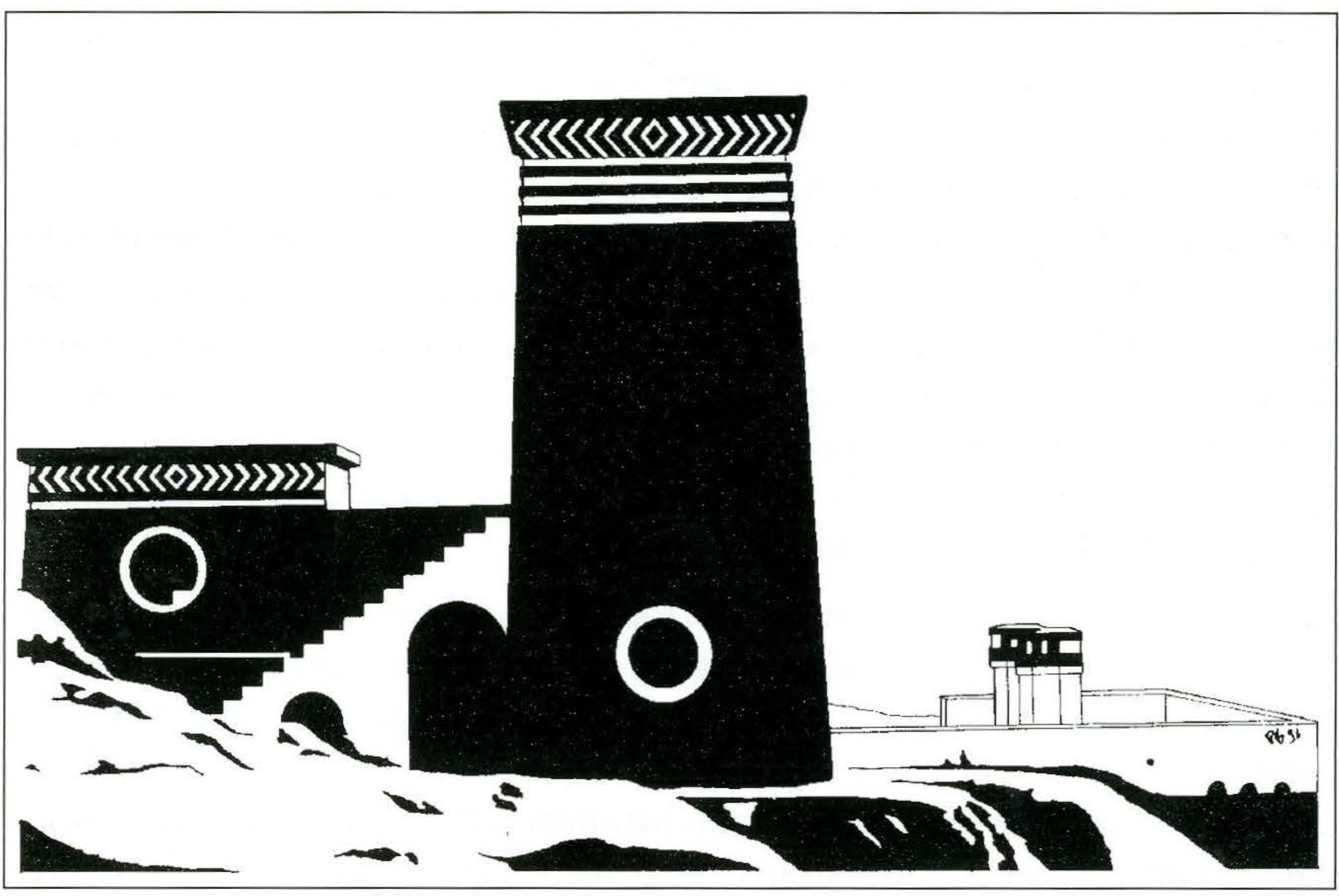

Station de pompage de Adram Sedum. D'après Onieva, Antonio J., Guía turística de Marruecos, Madrid, Artes Gráficas Argés, 1947, p. 496. Restitution : Pascal Carret.

fenêtres au premier étage. On a enfin quelques cas où la construction présente des corps flanqués de tours disposés symétriquement (façade de caserne à Axdir), mais ils constituent l'exception à ce qui apparait comme un véritable idéal d'asymétrie, au demeurant réussi.

Dans certaines œuvres, le style rifain, tout en demeurant fidèle aux normes fondamentales que nous avons décrites, présente des allures plus avant-gardistes et proches d'autres modèles d'un art déco aérodynamique à tendance plus cosmopolite. C'est très évidemment le cas de la station de pompage d'Adram Sedum où l'imposante masse rouge est rompue par des ouvertures en œil de bœuf (soulignées chromatiquement) et où l'escalier extérieur d'accès à la tour constitue un procédé architectural à fort effet visuel, suggérant l'idée d'une machine à large cheminée24. Cette même esthétique apparaît brillamment dans les bureaux des Intervenciones militares du Nekor, où le talus des parois extérieures ainsi que les fenêtres sont dérivées des formes d'un paquebot moderne. Bien qu'ayant en partie relégué ces premiers modèles d'esthétique rifaine, on peut également signaler une réalisation, la mahacina (poste de l'administration khalifienne) du souk de Beni Buaiach, où la structure du bâtiment est pleinement avant-gardiste : de son corps central surgissent ainsi deux avant-toits horizontaux, redevables d'un aérodynamisme ici fortement influencé par des volumes cubistes.

En guise de conclusion, on peut dire qu'un ultime regard sur l'architecture rifaine doit nous inciter à réfléchir sur cette solide tentative de créer un style nouveau, qui ne procédait pas de la copie de modèles antérieurs et qui porta la marque indé- 
lébile de l'originalité. La réflexion globale d'Emilio Blanco sur le Rif eut un caractère cohérent et diversifié, et son architecture en est indissociable ; elle représenta une sorte de compromis avec cette société, bien entendu dans le cadre immuable du colonialisme, ce qui détermina en fin de compte un style imposé d'en haut et dans des circonstances particulières. Cette réalité est à l'origine des contradictions de l'architecture rifaine, qui se matérialisèrent par son arrêt brutal au milieu des années 1940 et par l'oubli absolu dans lequel elle tomba par la suite, et qui conduisirent à une rapide "archéologisation" de ce qui, dans l'esprit de Blanco, devait être un puissant facteur de transformation du Rif.

\section{(Traduit de l'espagnol par Maria de los Angeles Rodriguez-Mellado et Mercedes Volait)}

\section{NOTES}

1. Le caractère nettement militaire de l'administration espagnole du Protectorat du Maroc deviendra une réalité à tous les niveaux. Dans un premier temps, on envisagea la figure du contrôleur civil, mais dès la fin des années 1920, la structure du Protectorat était entièrement formée par des militaires de différents corps et armes. Pour bien différencier les fonctions propres aux militaires de celles des contrôleurs, fut créée au cours des années 1920 une "Inspection générale d'Intervention Militaire et des Forces Khalifiennes" qui ne dépendait pas du ministère de la Guerre, mais désormais de la section des Affaires Indigènes du Haut Commissariat (López Oliván, 1931). NdT : nous avons choisi dans la suite du texte de traduire Interventor par contrôleur, mais de garder, pour son corps d'appartenance, la traduction plus littérale de "Interventions militaires".

2. NdT : Le terme de kabila (de l'arabe qabîla, tribu) désignait également, dans le vocabulaire colonial, une circonscription administrative correspondant globalement au territoire tribal préexistant.

3. Dans cette bibliographie, dont l'ouvrage de Rodolfo Gil Grimau (1982) donne un premier aperçu, méritent d'être distingués, entre autres, des auteurs comme Tomás García Figueras ou José Sánchez Pérez.

4. Les ingénieurs de l'armée avaient déployé une vaste et fructueuse activité architecturale dans toutes les villes du Protectorat (Tétouan, Larache, etc. ). Ils réalisèrent également une grande partie des extensions de Ceuta et Melilla, mettant en œuvre des typologies et des styles (comme l'art nouveau), qui étaient en principe du domaine réservé des architectes en titre. C'est pour tenter de corriger cette invasion irrégulière de compétences de la part des ingénieurs militaires que le Haut Commissariat allait créer le Service de l'Architecture.

5. En 1930, le Service de l'Architecture ne comprenait que trois architectes et deux conducteurs de travaux. Ils avaient à se partager la réalisation des projets et la conduite des chantiers de toutes les constructions dans le Protectorat.

6. On peut voir dans cette mise en garde de Vicente Martorell Otzet (qui était certes ingénieur de l'armée de réserve et possédait donc une bonne formation technique) un avertissement donné aux contrôleurs qui auraient pensé ou imaginé suivre l'exemple de Emilio Blanco, d'autant qu'elle intervint après le décès d'E. Blanco.

7. La zone du Protectorat espagnol offrait deux réalités bien différenciées : des régions fortement urbanisées, avec une population appartenant à l'ethnie arabe ou berbère arabisée, et d'autres absolument rurales où les berbères (rifains) avaient conservé des systèmes originaux d'organisation sociale et politique, ainsi que leur langue vernaculaire de la famille tamazight, le tarifit.

8. Significative, en ce sens, est l'identification revendiquée par un écrivain espagnol reconnu de la "Génération de 98" (une génération littéraire particulièrement prestigieuse en Espagne et profondément mar- 
quée par l'idée de regénération nationale), tel que José Martínez Ruiz "Azorín", qui devait ainsi écrire : "Moi aussi, je suis un peu berbère " (Bravo Nieto et Moga Romero, 1994).

9. Cet intérêt berbèriste favorisa l'étude de divers aspects de sa civilisation, tant de sa langue que de ses coutumes et de sa culture. Il transparait clairement dans bien des articles de la Revue Rifaine, éditée à Melilla (dès les années 1920, le "Tamasiit" y fut parfaitement reconnu comme langue, et différentes transcriptions de poésies y furent publiées) tout comme dans la publication par le père Esteban Ibañez (1944) d'un dictionnaire espagnol-rifain.

10. Pour Emilio Blanco, la société rifaine était à prédominance laïque et non religieuse. De ce fait, elle établissait un lieu de paix qui était le souk et non pas la mosquée, contrairement à ce qui se passait dans la société arabe (Blanco Izaga, 1939, 149) ; pour Blanco, le jour de paix rifain se devait d'être un jour séculier, commercial et "phénicien".

11. Selon Alfonso de Sierra Ochoa, Emilio Blanco prétendait ainsi offrir aux rifains des modèles architecturaux d'habitat concentré, face à la dispersion existant traditionnellement. Mais à la longue, cette éventuelle prétention ne dépassa pas un stade purement théorique. Alfonso de Sierra soutenait que Blanco « avait offert aux rifains... le début d'une unité politique à travers un style architectural » (Sierra Ochoa, 1951, 145). Cet architecte et écrivain fait une lecture clairement politique de l'architecture rifaine, en mettant en relation sur le plan des objectifs le nouveau style avec un peuple rifain que l'on espérait modeler dans le futur.

12. Le texte cité d'Alfonso Ochoa (1951), qui reprend une conférence faite à des futurs contrôleurs et qui parut après le décès de Blanco, constitue une remarquable exception de ce point de vue.

13. De nombreux spécialistes de la littérature coloniale hispanique ont dû se sentir visés par la plume sincère mais peu diplomate d'Emilio Blanco qui les traitait de "rats de bibliothèque" et de "scaphandriers de l'histoire" (Blanco lzaga, 1946, 315)

14. A la tête de la défense du style néo-arabe "cubiste" ou moderne se trouvait en Espagne, parmi d'autres, Rodolfo Gil Torres (1928) qui signalait la nécessité d'adopter ces formes esthétiques en ayant recours à des volumes simples et purs.

15. La ville de Melilla possédait un statut spécial dans le contexte nord-africain puisqu'elle constituait un territoire de souveraineté espagnole; ce fait individualisait radicalement son architecture et son urbanisme face à d'autres villes. En revanche un prestigieux architecte et écrivain espagnol, Leopoldo Torres Balbás (1923), critiquait avec acrimonie l'exportation de modèles européens dans des contextes nord-africains.

16. Dans ce contexte art déco et à cette même époque, naissait dans cette ville le dramaturge Fernando Arrabal.

17. L'architecture rifaine vernaculaire n'avait, selon Blanco, aucun style. Les descriptions de la façon dont les maçons rifains fabriquaient un mur sont réellement cocasses : « le mur n'est pas une œuvre d'art qui sort patiemment du cerveau et des mains de l'artiste qui le contemple ravi, mais un ennemi qui jaillit entre ses pieds et qu'il essaye de battre à coups de pierres, à coups de pieds et à coups de mains ", ce qui lui évoquait la « sauvage lutte primitive de l'homme contre la nature », (Blanco Izaga, 1930, 17).

18. En revanche, Blanco croyait trouver dans ces habitations grossières et primitives la "maison unique et la maison type" capable de s'adapter à toutes les fortunes et à toutes les activités, avec des intérieurs qui seraient désirés par les européens à cause de leur sobriété, de leur rationalité, de leur utilité et de leur confort ; en même temps, il critiquait férocement les typologies du logement européen qu'il estimait absurbes (Blanco Izaga, 1930, 27-35).

19. Cette architecture de Ksars du Sud Marocain était bien connue dans les années 1930, et Emilio Blanco disposait d'abondantes planches sur celle-ci (Sierra Ochoa, 1951, 145).

20. Nous avons pu retrouver certains de ces dessins dans les Archives du Génie à Melilla. (Sec. Technique, Dos. Su s/f) ainsi qu'un projet de guérite, non signé, très grossièrement dessiné en style rifain et dont nous n'adjugeons pas la paternité à Blanco à cause de sa médiocre facture, mais qui allait être un des canaux par lequel l'architecture rifaine allait s'imposer et se diffuser. 
21. Plusieurs entrepreneurs travaillèrent aux différentes constructions qui furent menées à bien dans le Protectorat ; certains se spécialisèrent dans ce type de travaux, comme ce fut le cas de Joaquín Burillo à qui l'on doit la construction de divers bureaux des Intervenciones Militares, tel que celui de l'Had de Ruade, du souk el Jemis ou de celui de Ketama. Ce fut aussi le cas d'autres entrepreneurs tels Juan Florido ou Lázaro Torres, tous originaires de Melilla.

22. Francisco Hernanz était un partisan convaincu de l'art déco aérodynamique. Des traits de cette nature "machiniste" et quelque peu avant-gardiste apparaissent dans des bâtiments rifains, telle la station de pompage d'Adram Sedum ou dans une mahacina (poste et bureau de l'administration khalifienne) d'un souk de Beni Urriaguel. Il est possible que ces traits aérodynamiques aient été suggérés à Blanco et qu'il les ait ainsi insérés dans son projet ou bien qu'Hernanz ait été amené à projeter ses propres œuvres dans les limites imposées par le style rifain.

23. Selon Blanco, l'ultime justification de cette tour et sa finalité étaient de permettre la lecture des récits d'Edgar Allan Poë pendant les nuits froides et venteuses du Rif (Sierra Ochoa, 1951, 147).

24. Un tel procédé n'est pas sans suggérer également une possible référence à l'architecture nubienne : dans certaines mosquées de Haute-Egypte, l'on retrouve de fait des dispositions analogues pour l'accès aux minarets, ainsi que me l'a signalé Mercedes Volait.

\section{BIBLIOGRAPHIE}

BLANCO IZAGA, Emilio (1930), La vivienda rifeña, Ensayo de característica e interpretación con illustraciones del autor, Ceuta, s. e., 61 p.

Blanco IZAGa, Emilio (1939), El Rif(2a parte) : La ley Rifeña II, Los Cánones Rifeños, Ceuta, Imprenta Imperio, $149 \mathrm{p}$.

BlanCO IZAGA, Emilio (1946), "Las danzas rifeñas, I, II y III", Africa, n 49/50, $56 / 57$ et 59/60, Madrid, janv./fév., août/sept., nov./déc. 1946, p. 315-316, 414-419, 547-551.

Bravo Nieto, Antonio (1991), "Europeismo y africanismo : dos ejemplos de arquitectura española del siglo XX en Marruecos", Boletín de Arte, n 12, Malaga, Universidad, p. 255-277.

Bravo NiETO, Antonio (1993), "El teatro como símbolo arquitectónico, Melilla y Tánger en torno a 1911 : Modernismo y Sezeción", Arquitectura y Ciudad III, Madrid, Instituto de Conservación y Restauración de Bienes Culturales, 1993, p. 350-364.

Bravo Nieto, Antonio et Moga Romero, Vicente (1994), "La contribution espagnole à la connaissance de la société marocaine coloniale...", in Actes du colloque Le Maroc oriental d'hier à aujourd'hui, Faculté des Lettres et des Sciences Humaines d'Oujda, 20 mai 1994 (sous presse).

CASAS MORA, Juan (1948), "Política", in Conferencias desarrolladas en la Academia de Interventores durante el curso 1947, Tetuán, Delegación de Asuntos Indígenas, p. 134-154.

Franco Bahamonde, Francisco (1925), "Política beréber", Revista de Tropas Coloniales, $n^{\circ} 3$, Ceuta, mars 1925, p. 6-7.

Franco BaHAmonde, Francisco (1926 a), "El principio del fin", Revista de Tropas Coloniales, $\mathrm{n}^{\circ} 18$, Ceuta, 1926, p. 121.

Franco Bahamonde, Francisco (1926 b), "Facetas del Protectorado", Revista de Tropas Coloniales, n² 23, Ceuta, décembre 1926, p. 265-266.

GIL GRIMAU, Rodolfo (1982), Aproximación a una bibliografia española sobre el Norte de Africa, 1850-1980, I, Madrid, Ministerio de Asuntos Exteriores, 869 p. 
GIL TORRES, Rodolfo (1928), "La vivienda moderna, Expresionismo árabe andaluz", Revista de la Raza, n 155/156, Madrid, juin/juillet 1928, p. 23-26.

HaRT, David M. (1958), "Emilio Blanco Izaga and the Berbers of the Central Rif", Tamuda, Tetuán, 1er semestre 1958, p. 171-237.

HART, David M. (1975), Emilio Blanco Izaga, Colonel on the Rif: a selection of his material, published and unpublished, on the sociopolitical structure of the rifians of Northern Morocco, New Haven, Connecticut, Human Relations Area Files, 2 vol.

IBAÑEZ, Fr. Esteban (1944), Diccionario Español-Rifeño, Madrid, Ministerio de Asuntos Exteriores, $440 \mathrm{p}$.

LEPRUN, Sylviane et SinOU, Alain (1984), Espaces coloniaux en Afrique Noire, Paris, UP nº 6, p. 274-345.

LOPEZ Olivan, J. (1931), Legislación vigente en la zona de Protectorado español en Marruecos recopilada y anotada por..., Madrid, Gráficas Marinas.

Martorell Otzet, Vicente (1950), "Las Obras Públicas y el Interventor", Selección de Conferencias y trabajos realizados por la Academia de Interventores durante el curso 1949-50, Tetuán, Delegación de Asuntos Indígenas, p. 185-195.

Perez Rojas, Javier (1990), Art déco en España, Madrid, Cáredra, 645 p.

SIERRA OCHOA, Alfonso de (1951), "Una teoría de arquitectura política y un Interventor excepcional : El coronel don Emilio Blanco Izaga”, Selección de Conferencias y trabajos realizados por la Academia de Interventores durante el curso 1950-51, Tetuán, Delegación de Asuntos Indígenas, p. 133-149.

TORRES BALBAS, Leopoldo (1923), "La arquitectura española en Marruecos”, Arquitectura, vol. V, Madrid, p. 139-142.

VOLAIT, Mercedes (1988), L'architecture moderne en Egypte et la revue Al-Imara (1939-1959), Le Caire, CEDEJ, 1988, 141 p.

VOLAIT, Mercedes (1992), “Architectures de la décennie pharaonique en Egypte (1922-1932)”, Images d'Egypte : de la fresque à la bande dessinée, Le Caire, CEDEJ, p. 163-186. 


\section{Figures de l'orientalisme en architecture}

Sous la responsabilité de Catherine Bruant, Sylviane Leprun et Mercedes Volait

Avec Dès les premières décennies du XIX ${ }^{\mathrm{c}}$ siècle, en un

A. Arrif mouvement que l'expansion coloniale allait accélérer,

A. Bravo Nieto le Maghreb et le Moyen-Orient ont représenté un

S. Burth-Levetto important terrain d'activité pour les professionnels

J.C. David européens de l'architecture et de l'urbanisme. Leurs

F. Friès

A. Gerber

M. Ghorayeb

X. Malverti

M. Marié

N. Montel

N. Oulebsir

B. Pagand

E. Pflugradt-Abdel Aziz savoir-faire se sont exercés à des échelles et dans des domaines variés : de la construction de bâtiments isolés à l'aménagement du territoire, en passant par la fondation de villes, l'extension de cités existantes, le remodelage de centres anciens, ou encore l'inventaire et la préservation des patrimoines locaux. Il en est résulté un vaste ensemble de réalisations où des répliques de formes européennes côtoient les métissages de styles

A. Picard

P. Pinon

M. Sakka-Thiveou

P. Tsakopoulos

A. Van Loo les plus audacieux. Confrontés à des réalités techniques, sociales et culturelles différentes, qui n'étaient pas sans les fasciner, ces hommes de l'art n'ont pu dans bien des cas y être imperméable. Ils trouvèrent aussi en ces territoires un espace d'innovation et d'expérimentation, qui ne fut pas toujours sans effet sur l'évolution des cultures techniques et artistiques métropolitaines. C'est à explorer ces différents phénomènes d'échanges et d'interactions et à tenter d'en mieux cerner la réalité, à partir de sources inédites, que s'attachent les différentes études réunies dans le présent volume.

\section{Prochaines LiVRaISONS}

$\mathrm{N}^{\circ} 75$ : Oral et écrit dans le monde turco-ottoman (sous la responsabilité de Nicolas Vatin).

$\mathrm{N}^{\circ} 76$ : L'humour dans l'empire ottoman (sous la responsabilité de François Georgeon).

$\mathrm{N}^{\circ}$ 77-78 : Biens collectifs et gestion communautaire dans les mondes musulmans (sous la responsabilité de Sylvie Denoix).

Illustration de couverture : Médersa construite à Alger, en 1905, par Henri-Louis-Paul Petit (1856-1926). Collection S. Leprun.

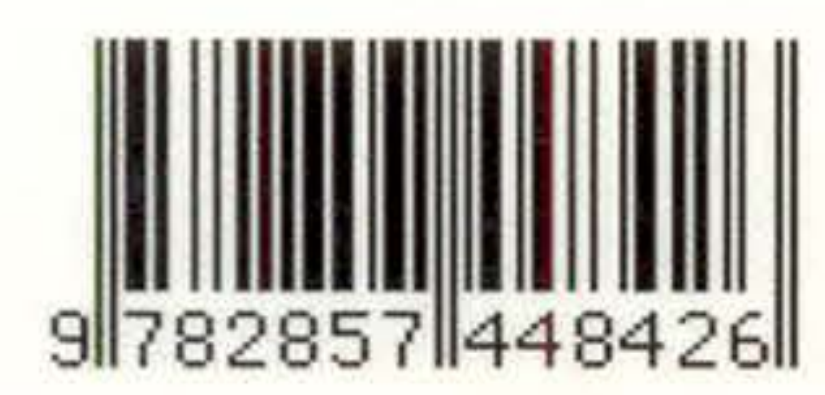

